\title{
Une proposition de mutation des pratiques de lecture mise en échec : le Cybook de Cytale, 1998-2002
}

A Failed Proposal to Change Reading Habits: Cytale's Cybook, 1998-2002

Dominique Nauroy

\section{OpenEdition}

\section{Journals}

Édition électronique

URL : http://journals.openedition.org/edc/407

DOI : $10.4000 /$ edc. 407

ISSN : 2101-0366

Éditeur

Université Lille-3

Édition imprimée

Date de publication : 1 décembre 2006

Pagination : 157-171

ISBN : 2-9514961-7-6

ISSN : $1270-6841$

Référence électronique

Dominique Nauroy, «Une proposition de mutation des pratiques de lecture mise en échec : le Cybook de Cytale, 1998-2002 », Études de communication [En ligne], 29 | 2006, mis en ligne le 19 janvier 2009, consulté le 03 mai 2019. URL : http://journals.openedition.org/edc/407 ; DOI : 10.4000/edc.407

Ce document a été généré automatiquement le 3 mai 2019.

() Tous droits réservés 


\title{
Une proposition de mutation des pratiques de lecture mise en échec : le Cybook de Cytale, 1998-2002
}

\author{
A Failed Proposal to Change Reading Habits: Cytale's Cybook, 1998-2002
}

Dominique Nauroy

\section{Introduction}

Dans le domaine des industries culturelles, les vingt dernières années ont été fertiles en innovations, réussies ou ratées. On peut considérer, notamment à l'instar de Patrice Flichy, que les échecs sont aussi intéressants pour l'observateur que les réussites. Pourtant, trop rares encore sont les ouvrages qui, tel Aramis ou l'amour des techniques de Bruno Latour, procèdent à l'autopsie d'un échec technologique. Prenant appui sur la sociologie de la traduction, cet article se propose de revenir en détail sur les conditions de l'échec de Cytale, la société française qui a conçu et tenté de diffuser au début des années 2000 un livre électronique, outil de médiation culturelle, le Cybook.

2 Fondée par Jacques Attali, en 1998, Cytale s'est emparée d'un champ de l'industrie culturelle qu'aucun acteur n'avait encore tenté de faire migrer vers le monde numérique : celui de la lecture. Prenant acte d'une part du succès de la dématérialisation de la musique, d'autre part des modèles de vie nomade dont Jacques Attali prédisait l'essor rapide, Cytale propose en 2001 une tablette de lecture électronique, le Cybook, destinée à un segment de marché grand public constitué de grands lecteurs, d'expatriés, de seniors et de voyageurs. Comment Cytale a-t-elle tenté d'en publiciser et d'en justifier l'usage auprès de ses publics?

Quelques années auparavant, Jacques Attali avait notamment soutenu ${ }^{1}$ que nous allions nous percevoir de moins en moins comme des sédentaires et de plus en plus comme des 
nomades. Des présentations de Cytale, qui datent de février 2000, estimaient de ce point de vue que les comportements avaient effectivement évolué. Le «nomade » faisait partie du panel idéal des clients que Cytale avait défini peu avant le lancement commercial de sa solution. Le Cybook était une tablette électronique de lecture, qui devait l'accompagner en lui offrant " une bibliothèque en permanence mise à jour $"^{2}$ : selon Jacques Attali, le livre électronique correspondait à une « nécessité culturelle $»^{3}$.

Pourtant, sa commercialisation fut un échec. Dès le lancement de sa campagne publicitaire, Cytale ne s'y est pas trompé : alors pionnière et solitaire sur un marché nouveau, elle savait que l'adversaire ne serait pas un artefact technologique plus audacieux, mais le livre imprimé lui-même, dont le Cybook tentait de mimer les propriétés, afin de garder intactes les conditions de présentation du texte sur la page, et de respecter les habitudes de lecture. Pourtant, le livre électronique est vu comme un ennemi par bon nombre d'auteurs ${ }^{4}$, un pis-aller par une majorité d'éditeurs ${ }^{5}$, il s'agissait donc de s'afficher comme une solution complémentaire, et non pas concurrente, respectueuse des canons de l'écriture romanesque, des standards multi centenaires de présentation du texte sur la page ainsi que des circuits de diffusion des œuvres. En dépit de réels exploits techniques, légitimer la filiation du Cybook par rapport au livre dans la sphère publique serait le travail le plus acharné des membres de Cytale.

\section{La proposition de Cytale}

5 Par le truchement d'une tablette électronique dédiée à la lecture de romans français contemporains, Cytale veut proposer un nouvel accès à la lecture, et non une nouvelle approche de la lecture. Le projet de Cytale n'est pas de nous inviter à lire autrement, mais plutôt à lire plus, de rendre plus aisé l'accès à la lecture. Cytale va s'appliquer, avec un soin dont la méticulosité dépasse quelquefois la mesure, à respecter les conventions de lecture.

Mais le Cybook se présente comme une traduction inadaptée aux yeux des publics auxquels Cytale s'adresse. De lui-même, comme en témoignent de dramatiques microtrottoirs, le Cybook n'est pas éloquent: est-ce un livre, est-ce un ordinateur? Les distributeurs, qui ont un rôle déterminant dans la perception des dispositifs et dans la reconnaissance de leurs qualités, éprouvent certaines difficultés à positionner ce produit, qui n'a sa place nulle part, ni en librairie, ni au rayon des PDA, ni même indépendamment au sein d'un « linéaire » e-book qui n'aurait de sens que dans le cas d'une concurrence.

7 La carte "grand public» permet d'intégrer au capital de l'entreprise de nouveaux actionnaires, venus défendre ce choix, mais le public, exigeant, pose des questions sur la nature et l'utilité de la tablette. Nombre des titres disponibles, coût de la tablette, objet porteur d'une tendance en conflit avec le livre papier qui, lui, revendique une âme face à l'objet technique, froid et inerte : voici quelques thèmes que Cytale doit traiter face à un public qui a le choix entre une tablette et son rival le livre, un « monstre sacré » dont on prédisait la mort à la fin du siècle dernier et qui, en réalité, affiche une santé de fer. Le Cybook est-il «compatible culturellement»? Vu les performances fonctionnelles qu'il offre et l'économie de moyens qu'il permet, certains considèrent le livre papier comme un summum technologique absolu, vraisemblablement indépassable. La tablette, elle, n'a pas d'épaisseur et elle réduit aux seules dimensions de l'in-octavo les divers formats des ouvrages. Le livre électronique fait perdre la notion d'espace et de livre classé dans une bibliothèque qui, elle-même, suivant l'endroit où elle se trouve, peut avoir un sens ${ }^{6}$. Le 
livre papier recèle en lui des valeurs affectives et sociales qui dépassent le seul intérêt porté au contenu. Il existe entre un livre et son lecteur une relation particulière et complexe, où interviennent la qualité du papier, son odeur, les caractéristiques de la couverture, de la typographie. On peut parcourir un livre avant de l'acheter : c'est une opération déjà plus délicate dans le cadre d'un livre numérique. Enfin, les nomades déplorent le poids de la tablette électronique : «Tout sauf une invitation au voyage " ${ }^{7}$. Ce sont des aspects qui ont une valeur d'analyse uniquement parce que Cytale a nourri un dessein grand public. Et ce n'est que sous cet éclairage que l'on peut comprendre les jugements sévères de la presse sur une solution considérée comme "un joujou dispendieux $»^{8}$.

8 Dans l'attente, malheureusement jamais satisfaite, d'une concurrence, le modèle commercial de Cytale est un "tout" à prendre ou à laisser, incluant des œuvres numérisées et formatées à destination d'une tablette électronique capable de les présenter aux yeux du lecteur. En France, Cytale reste seul fournisseur d'œuvres littéraires numérisées à destination de livres électroniques, et surtout la seule entreprise qui incarne le livre électronique : l'absence de concurrence inspire au public beaucoup d'hésitation face à une solution fermée et qui engendre trop d'inerties.

9 L'échec commercial, dont le passage par le circuit FNAC reste emblématique, invite à reconsidérer le modèle de diffusion. L'innovation est un acte de foi, souligne Norbert Alter dans L'innovation ordinaire, et c'est un domaine où aucune étude d'aucun ordre, si rationnelle qu'elle fût, n'aurait pu donner une indication précise sur l'acceptation du produit par le marché. Le succès d'un nouveau produit, tel le Cybook, n'est que très faiblement prévisible. Si Cytale a été seule à commercialiser un livre électronique en France, elle n'a pas été seule à croire en l'expansion rapide des tablettes de lecture électroniques autorisant à lire des livres numériques. Avant de reconnaître rapidement leur excès d'enthousiasme, les cabinets d'analyse avaient promis dans un premier temps de florissants marchés aux pionniers partis à l'aventure dans ce secteur. Cytale n'a pas été la seule à affirmer en 2000 que le modèle du livre électronique était «viable techniquement, économiquement, socialement». En réalité, le livre électronique est moins une innovation qui a raté un marché existant qu'une innovation qui n'a pu prendre le temps de bâtir son propre marché.

10 Ainsi, l'engouement général autour d'une invention nouvelle contribue à expliquer le choix initial de segments de marché grand public, et la focalisation trop tardive sur un marché plus captif, le monde du handicap.

\section{Un compromis négocié avec les éditeurs et imposé au public}

11 Utiliser l'électronique pour parvenir à séduire les amoureux de la lecture (qui, bien souvent, ne sont pas des amoureux de la technique, alchimie rare qu'a réussi à produire Cytale en son sein) peut sembler une gageure : en 2000, personne ne conçoit a priori le livre électronique comme une invitation à la lecture. De sorte que, pour certains publics, la frustration est double : d'une part, ce n'est pas encore un livre; d'autre part, bien qu'il en possède les propriétés techniques, ce n'est plus un ordinateur, car le Cybook interdit l'accès à une autre interface que celle du logiciel de lecture. 
12 Mais Cytale traduit d'abord les intérêts des éditeurs français, dont « l'enrôlement » est un préalable indispensable au développement d'une solution de diffusion numérique des œuvres littéraires. C'est en tout cas dès les premières heures la conviction forte de Cytale, qui préfère les intégrer à une dynamique commune et gagner une légitimité auprès d'eux en s'affichant comme un partenaire proche, respectueux de leurs intérêts. Et, du coup, on ne peut comprendre la succession des actions et événements qui ont émaillé l'histoire de Cytale qu'à la condition de les relier à ce lien qu'elle s'est appliquée à créer, et qu'elle va sans cesse développer. Le projet de Cytale doit rester justifié en des termes qui demeurent cohérents avec des objectifs jugés légitimes pour les éditeurs.

Michel Callon rappelle ${ }^{9}$ 'étymologie du verbe " intéresser » : se placer entre (inter-esse en latin), s'interposer. La stratégie de légitimation de Cytale auprès des éditeurs l'amène d'abord à desserrer les liens que d'autres entités concurrentes (Microsoft et Gemstar, par le biais de la maison d'édition en ligne 00h00) s'efforcent d'établir avec eux. Le dispositif d'intéressement de Cytale construit un système d'alliances par opposition aux solutions tierces présentées comme dangereuses pour l'éditeur. Dans cette manche qu'elle remportera, Cytale part désavantagée car moins au fait des us et réseaux d'un monde que connaît en revanche très bien 00h00, start-up que dirige l'ancien PDG de Flammarion, Jean-Pierre Arbon. Mais Cytale se crée une position originale, et surtout non concurrentielle, en se définissant comme diffuseur numérique et non comme éditeur numérique. C'est guidée par ce même souci de pas nuire aux éditeurs et à leur réseau de distribution, c'est-à-dire essentiellement aux libraires, que Cytale n'usera jamais de comparaisons qualitatives en faveur de la tablette électronique par rapport au livre.

Malgré l'évidente fragilité de la jeune start-up et l'éventualité du rachat complet ou partiel de son activité par l'un des deux pôles éditoriaux majeurs ou un partenaire industriel inconnu, les éditeurs acceptent de confier leurs titres à Cytale, qui en assure la diffusion sur son propre réseau sécurisé. Alliée des éditeurs, Cytale défend la position difficilement tenable d'un dispositif fermé, seul capable de rendre impossible la lecture d'ouvrages piratés. Le Cybook est du coup un artefact taillé pour les éditeurs, devenant d'une part le vecteur exclusif de la production littéraire contemporaine dans le monde numérique, d'autre part un dispositif monocanal (plusieurs éditeurs, mais un seul type de source) et mono-usage.

Cytale s'interdit de faire évoluer les fonctions et usages de son dispositif et, plus grave peut-être, interdit à ses publics de créer des usages non prévus et qui pourraient devenir majoritaires, jusqu'à constituer des arguments phares en faveur du Cybook. En interdisant à ses clients d'accéder au fichier littéraire autrement qu'en le lisant sur la tablette, Cytale offre une garantie de sécurité aux éditeurs, et s'assure une garantie de revenus. D'elle-même, elle s'interdit d'être éditrice, évitant de reproduire des précédents américains que redoutaient également les maisons d'édition. Ainsi, la clarté avec laquelle Cytale définit et sélectionne les sources d'où proviennent les contenus annule la méfiance liée à la qualité très fluctuante des textes que l'on trouve alors en numérique, comme le soulignent Roger Chartier ${ }^{10}$ et Umberto Eco ${ }^{11}$.

\section{Bâtir la légitimité de la lecture électronique}

Le premier semestre 2000 voit se dérouler un combat singulier, par le biais de tribunes médiatiques, entre les preux défenseurs du livre papier qui crient au sacrilège et à la 
duperie, et quelques plumes ferventes qui ont hâte de s'affranchir des contraintes jugées rédhibitoires du vieux codex. Cytale entre presque seule dans un débat qui consiste à savoir si l'écran ne fait pas précisément "écran» au roman, empêchant notamment l'accès par feuilletage ou la découverte d'une fleur séchée entre deux pages. Elle propose un medium de substitution, et laisse passer l'occasion de tirer profit d'œuvres spécifiques auxquelles le Cybook, par différence avec le livre imprimé, aurait pu donner accès. Cytale va jusqu'à refuser, pour sa communication promotionnelle, des propositions de slogans tels que: "Cybook, vous n'aviez jamais lu comme cela", car il s'agit d'afficher sa complémentarité, et non sa différence ; de rester humble, et non de prétendre proposer mieux; de clamer une filiation avec le livre, et non de proposer une expérience radicalement nouvelle. Guidée par un même souci de pas nuire aux éditeurs et à leur réseau de distribution, c'est-à-dire essentiellement les libraires, Cytale choisit de ne pas capitaliser sur le pouvoir distinguant de son objet.

Des équipes de recherche scientifique viennent aider Cytale à bâtir la légitimité de la lecture électronique. L'enquête "Contrats de lecture $»^{12}$ que coordonne Claire Bélisle de l'ENSSIB officialise les premiers rapports d'usage entre l'objet livre électronique et l'usager en France. Elle indique notamment que la tablette de lecture dédiée, qui prend en compte l'ergonomie visuelle et le confort du lecteur, fait disparaître l'incompatibilité entre le plaisir de lire et l'écran. Le Cybook est alors en train de devenir un objet social à travers l'appropriation de l'usager, malheureusement trop tard dans la courte histoire de l'entreprise pour que Cytale puisse en tirer profit.

\section{Les publics de Cytale considèrent l'invention sous un œil différent}

18 L'échec de sa proposition amène Cytale à renégocier sa solution à destination du public des malvoyants. Grâce à son système d'agrandissement de caractères, le Cybook est, dans ce cas, un artefact qui donne un accès nouveau et véritablement unique à un univers culturel. Plus que de mutation, il convient alors de parler de création de nouveaux usages. Rappelons le postulat de départ : l'innovation que constitue le Cybook donne à lire à tous ceux qui manquent de lecture, c'est-à-dire ceux qui, pour des raisons pratiques ou culturelles, n'ont pas un accès commode au livre.

Cytale agrandit son réseau aux malvoyants, tout en se trouvant dans l'obligation absolue de maintenir l'union de ses premiers alliés. La société se repositionne sur une niche pour laquelle son innovation constitue, de son point de vue, une condition quasi sine qua non de l'accès à l'écrit. L'intérêt de ce marché pour Cytale a fait l'objet d'une réelle controverse lors des entretiens menés post mortem avec les acteurs de Cytale. Le Cybook était conçu dès le départ pour afficher le texte d'une œuvre en différentes tailles, exigence qui impacte le travail de l'équipe chargée du formatage numérique des œuvres. Mais son interface se présentait comme une traduction inadaptée au besoin d'un malvoyant d'accéder, seul et en toute autonomie, à l'œuvre. Une négociation a permis de concevoir un dispositif accepté et reconnu par la population à laquelle il se destine.

21 Si la proposition de Cytale pouvait, ici, être opérante, c'est parce que les dispositifs dédiés au handicap visuel sont rares et se résument à des appareils de grossissement chers et peu commodes ou à une sélection de textes édités en corps 16. C'est donc un marché demandeur et qui, par la voix de ses porte-parole, demandait depuis longtemps aux 
éditeurs des versions numérisées des romans contemporains. Jusqu'à l'arrivée du Cybook, pour des problèmes de sécurisation des contenus, les éditeurs n'avaient pas souhaité répondre positivement à cette demande.

Cytale crée ainsi une médiation entre des acteurs qui, jusqu'alors, n'avaient pas trouvé une formule adéquate sur laquelle s'entendre. D'abord innovation destinée à une certaine "élite ", le Cybook tend ensuite à corriger une inégalité sociale face à la lecture. Le problème que Cytale doit résoudre ici est, en apparence uniquement, d'un autre ordre : autant ses premières cibles étaient hétérogènes et vastes, donc difficiles à cerner, autant la malvoyance constitue une cible très précise, mais en réalité dispersée, anonyme, à laquelle il est donc difficile de s'adresser.

La manière selon laquelle Cytale a été amenée à reconsidérer ses publics est intéressante à plus d'un titre :

- Les résistances auxquelles la société doit faire face mettent en évidence le statut référentiel du livre imprimé dans l'imaginaire collectif. D'où une inadéquation entre l'œuvre romanesque et l'écran, en dépit d'expérimentations réalisées en bibliothèques (ENSSIB, 2002) prouvant le contraire.

- Malgré la pertinence du choix comme cible d'un public malvoyant, qui se confirme mois après mois, Cytale continue d'adresser sa communication au grand public, pour ne pas véhiculer immédiatement l'image d'un produit destiné au traitement d'un handicap. Cette politique marketing est le résultat d'un désaccord au sein du conseil d'administration, dont une partie continue de croire à la validité des premières cibles. Pourtant les vecteurs d'opinions, d'abord très favorables au concept, se retournent brutalement en l'espace de quelques mois ${ }^{13}$.

- La stratégie d'offre de Cytale peut se résumer à l'évolution de ses publics : elle passe de la proposition d'une mutation des pratiques de lecture, qui se heurte à un adversaire de taille, à la création de nouvelles pratiques de lecture. Ou d'une invention "dogmatique ", pour reprendre les mots de Norbert Alter, peu malléable, interdisant à son public de l'adapter à de nouveaux usages, commercialisée selon un modèle diffusionniste, à une solution sine qua non négociée. Cytale crée, ici, une médiation réussie.

\section{Survivre en négociant les usages du Cybook avec ceux qui lui ont conféré une légitimité}

Le grand public ne le trouve pas nécessaire, les malvoyants sont, à l'opposé, captifs mais difficilement accessibles. Ce qui est clair, c'est que le livre électronique doit d'abord être un produit de niche, comme le note la presse au cours de l'année $2001^{14}$. Et dans ce cadre, les bibliothèques deviennent les relais idéaux d'une telle innovation au sein d'un plus large public, essentiellement constitué de grands lecteurs.

Bibliothèque nomade, le Cybook pourrait facilement se concevoir comme un ennemi des bibliothèques telles que nous les connaissons. Il est frappant de constater à quel point cela a pu être l'inverse, dans le cas de Cytale comme de Gemstar, SoftBook Press ou Nuvomedia aux États-Unis. Assez rapidement, certains bibliothécaires acceptent de revoir la définition de leur mission, qui se déporterait progressivement du métier d'acquéreur à celui de négociateur de contrats d'accès, pour citer Pierre Le Loarer ${ }^{15}$ ou, plus récemment, Patrick Bazin ${ }^{16}$, directeur de la bibliothèque de Lyon. 

place par Cytale se caractérise à la fois par la non-malléabilité de l'œuvre, alors que la nature même du numérique la rendrait possible, et par la non-malléabilité de la machine, qui empêche toute transgression de l'usage canonique. Pourtant, le succès d'une innovation peut se trouver dans les pratiques non prévues par les concepteurs, et que le dispositif pourrait toutefois encourager ou permettre, selon Jacques Perriault ${ }^{17}$ ou Norbert Alter ${ }^{18}$. Dans le domaine de la musique numérisée, l'iPod d'Apple autorise une évolution « lente » des logiques de consommation : il permet d'abord de faire perdurer les pratiques illégales avec lesquelles l'usager est familier, en l'accompagnant progressivement vers le modèle légal qu'Apple préconise. processus d'autolégitimation qu'avait initié Cytale auprès d'eux. Car Cytale n'a pas hésité à se servir de la peur du piratage, que tout autre dispositif, moins scrupuleux que la solution qu'elle promettait, aurait engendré. Devenue nécessaire aux yeux des éditeurs, cette solution devait ensuite tenir ses promesses, et nous comprenons ici pourquoi Cytale n'est pas parvenue à la renégocier pour l'assouplir en vue d'une utilisation plus aisée du Cybook en bibliothèques, ainsi que le notent Claire Bélisle et Christian Ducharme dans le rapport d'expérimentation de $2002^{19}$. Car rapidement, les bibliothèques se révèlent des clients intéressants et capables de faire connaître cette innovation auprès des populations que cible Cytale. Mais, pour reprendre les termes de l'école de la traduction, Cytale n'a pas su s'ériger en porte-parole représentatif à la fois des éditeurs et des bibliothécaires pour construire une solution commune qui, d'une part, aurait reçu l'aval de chaque «allié » mobilisé et qui, d'autre part, aurait sans doute garanti à Cytale sa survie. Le dispositif d'intéressement patiemment élaboré avec les éditeurs n'est pas adapté au fonctionnement des bibliothèques, et la proposition nouvelle qui vise à intégrer leurs intérêts risque de desceller les premières alliances, mouvement qui serait alors fatal à Cytale.

Car les bibliothécaires, «mobilisés » par le Cybook, souhaitent l'adapter à leur économie en brisant le lien unique établi entre un livre numérique acheté et la tablette sur laquelle il est censé être lu. Cette contrainte technique nuit au service de prêt, c'est-à-dire à la diffusion des œuvres. C'est ce que révèle une étude menée par l'Université de Rochester aux États-unis sur l'usage des livres électroniques en bibliothèques ${ }^{20}$. Comme l'illustrent Claire Bélisle et Christian Ducharme, dans le cas d'un usager qui désire lire une œuvre achetée avec une tablette qui est actuellement en prêt, la bibliothèque ne pourra pas satisfaire cette demande, même si l'œuvre n'a pas été chargée sur la tablette déjà prêtée ${ }^{21}$. Les bibliothécaires proposent alors le système d'une licence de diffusion, autorisant par exemple dix lectures, simultanées ou non, sur des tablettes différentes, d'une même œuvre. Ces nouveaux acteurs vont donc, par leur voix, tenter de proposer le mode de diffusion des œuvres auquel Cytale avait pensé de son côté, c'est-à-dire selon une logique d'accès : la circulation du fichier ou du livre numérique n'a plus d'importance, elle n'est plus contrôlée, c'est son accès qui doit être désormais régulé. Mais Cytale ne peut proposer cette nouvelle traduction sans remettre en cause une partie de la traduction sur laquelle il y avait eu précédemment accord. C'est contre la volonté de remettre en cause une première traduction du dispositif, encore à ses débuts, que les éditeurs s'érigent, criant à la trahison que constituerait cette nouvelle traduction.

29 L'échec de la solution tentée avec les bibliothèques met en lumière un désaccord profond entre deux logiques de distribution de l'œuvre : la propriété ou l'accès. Et on ne peut 
comprendre l'aventure de Cytale si on ne met pas en évidence sa tentative, pour assurer sa survie, de traduire des intérêts parfois opposés, mettant ainsi en cause la pertinence de son projet auprès de ses premiers alliés.

\section{Refuser la filiation?}

Comme tout produit nouveau, le Cybook est une invention qui a cherché sa voie et qui a dû apprendre à négocier ses formes et ses fonctionnalités avec les marchés pour lesquels il s'était découvert des atomes et des octets crochus. Et le conseil qui administrait Cytale ne s'est sans doute accordé que bien trop tard autour d'une traduction commune.

Un medium peut commencer par imiter ses prédécesseurs, mais ce constat a conduit certains acteurs, et notamment Jacques Attali, à revoir la stratégie qu'avait d'abord promue Cytale, pour, précisément, se démarquer du livre, et donc lire autrement. Au cours de la vie de Cytale, Jacques Attali a eu ainsi parfois tendance à s'éloigner de la ligne selon laquelle le Cybook facilite la même lecture immersive que le livre papier, en suggérant plutôt une lecture "d'île en île ", empruntant des " chemins bifurcatoires ", favorisée par l'hypertexte. Car Cytale reproduit la page, mais pas le livre, du moins pas intégralement. Le mimétisme voulu n'est pas totalement réalisé, alors pourquoi ne pas tirer profit des autres usages que le Cybook a la capacité de proposer? Trop occupée à légitimer la filiation de son invention avec le livre, Cytale inaugurera, peu de temps avant sa mort, ce qui aurait pu, sans doute, constituer la réelle valeur ajoutée du livre électronique et du livre numérique aux yeux du grand public. Marc Vasseur, père du projet, considérait alors son innovation comme " plus intéressante en termes de rupture ».

À cette époque, le choix suivant s'offrait à Cytale: favoriser la création d'œuvres originales dédiées au livre électronique, raconter les histoires d'une autre manière, trouver son propre langage de telle sorte que le livre papier devienne, ainsi que le prédit l'écrivain Alexandre Jardin, «le signe distinctif d'une classe sociale restreinte, enfermée dans sa bulle et déconnectée des modes d'expression moderne $»^{22}$. Ou proposer, en périphérie du texte proprement dit, un point de vue sur l'univers propre à l'auteur, adaptant ainsi la formule du DVD vidéo accompagné de bonus, avec le bémol que le marché de la littérature est moins concentré. C'est vers cette deuxième voie que Cytale se dirige, adaptant notamment La grammaire est une chanson douce, le livre d'Érik Orsenna.

\section{Conclusion}

C'est auprès du grand public que Cytale a le mieux défendu son " contrat de lecture » au cours de son existence. Mais c'est au service d'une niche de malvoyants que Cytale a travaillé avec le plus de constance. Et c'est en évoquant ce dernier public qu'après la mort de la société les responsables de Cytale tentent de justifier leur projet. Comme pour tout produit nouveau, le Cybook est une invention qui s'est cherchée et qui a dû apprendre à négocier ses formes et ses fonctionnalités avec les marchés avec lesquels il s'était découvert des atomes et des octets crochus. Mais le conseil qui administrait Cytale ne s'est sans doute accordé que bien trop tard autour d'une traduction commune. Il y eut en réalité autant de Cybooks qu'il y avait d'écoles de pensée au sein de ce conseil, parce qu'aucune n'a cessé de croire en son projet. De la sorte que, si nouveau qu'il soit lors de sa 
sortie commerciale en janvier 2001, le Cybook a cependant déjà une histoire, marquée par deux années de conception et de controverse interne. d'Amazon et de Google aux États-Unis dans le domaine de l'édition numérique, et de Sony au Japon dans le domaine d'un livre électronique d'un genre nouveau, un hybride qui mêle de manière convaincante les propriétés de la feuille et de l'écran.

\section{BIBLIOGRAPHIE}

Alter, Norbert, (2000), L'innovation ordinaire, P.U.F. sociologies, Paris.

Attali, Jacques, (1996), Chemins de sagesse : un traité du labyrinthe, Paris, Fayard.

Bélisle, Claire, (2002), Contrats de lecture, Rapport sur une expérimentation de prêt de livres électroniques en bibliothèques : dimensions technico-économiques et socio-cognitives, Lyon.

Bélisle, Claire et Ducharme, Christian, (2003), « Livres électroniques et bibliothèques municipales ", Bulletin des Bibliothèques de France, Paris, t. 48, n 3, pp. 74-86. 
Callon, Michel, (1986), « Éléments pour une sociologie de la traduction : la domestication des coquilles Saint-Jacques et des marins-pêcheurs dans la baie de Saint-Brieuc », L'Année sociologique, n³6, p.170-208.

Chartier, Roger, (2001), Lecteurs et lectures à l'âge de la textualité électronique, Paris, Bibliothèque publique d'information.

Le Monde, (13 mars 2001), « Quels lecteurs pour l'eBook?».

Le Loarer, Pierre, (14 mai 2002), « L'édition électronique : rôles des médiateurs et nouveaux rapports au savoir ", dans le cadre du $70^{\mathrm{e}}$ Congrès de l'ACFAS « Publications et lectures numériques : problématiques et enjeux », IEP Grenoble.

Mauger, G., Poliak, C. et Pudal, B., (1999), Histoires de lecteurs, Paris, Nathan.

Morel, Christian, (2002), Les décisions absurdes : sociologie des erreurs radicales et persistantes, Paris, Gallimard.

Perriault, Jacques, La logique de l'usage, Paris, Flammarion, 1989.

\section{NOTES}

1. Attali Jacques, Chemins de sagesse : un traité du labyrinthe, Paris, Fayard, 1996.

2. Extrait de l'interview de Jacques Attali diffusée dans l'émission Tracks sur Arte, le 17 octobre 2000.

3. Ibid.

4. Nous reprenons l'expression de l'écrivain américain Nick Tosches ( Tracks, Arte, 17 octobre 2000).

5. En 2000, Laurent Beccaria, patron de la maison d'édition Les Arènes, considère absurde le nom d'éditeur en ligne et juge sévèrement la qualité du catalogue de la maison d'édition en ligne $00 h 00:$ : Ce sont des livres traditionnels, des romans, des essais, qui ont été vraisemblablement refusés par $90 \%$ de la profession, qui ont été mis en ligne au format électronique et que vous pouvez télécharger sur votre ordinateur. Donc c'est le plus mauvais des livres sous la forme la plus pauvre qui soit. Et en plus on vous le fait payer ».

6. Mauger Gérard, Poliak Claude et Pudal Bernard, Histoires de lecteurs, Paris, Nathan, 1999.

7. Extrait d'un article paru dans Le Temps de Genève, 4 mai 2001.

8. On retrouve cette dépêche AFP de 2001 par exemple sur http://www.mmedium.com/cgi-bin/ nouvelles.cgi?Id=4775 ou sur http://www2.canoe.com/techno/nouvelles/ archives/2000/12/20001215-172410.html.

9. Michel Callon, «Éléments pour une sociologie de la traduction : la domestication des coquilles Saint-Jacques et des marins-pêcheurs dans la baie de Saint-Brieuc », L'Année sociologique, 1986.

10. Chartier Roger, Lecteurs et lectures à l'âge de la textualité électronique, Paris, Bibliothèque publique d'information, 2001.

11. Interview d'Umberto Eco par Gloria Origgi, Paris, Bibliothèque publique d'information, 2001.

12. Bélisle Claire, «Contrats de lecture, Rapport sur une expérimentation de prêt de livres électroniques en bibliothèques : dimensions technico-économiques et socio-cognitives ", Lyon, 2002. Lire également Bélisle Claire et Christian Ducharme, BBF 2003 - Paris, t. 48, n 3, pp. 74-86.

13. De « l'objet qui pourrait bien révolutionner le monde » (Capital, juin 2000), dont «l'avenir ne semble guère faire de doute » (Le Monde, décembre 1999) au « livre électronique [qui] n'a pas tenu ses promesses» (SVM, décembre 2001) en passant par «e-book: Gutenberg recalé en numérique " (Science et Vie, mai 2001).

14. Le Monde, «Quels lecteurs pour l'eBook?», 13 mars 2001. 
15. Le Loarer Pierre, "L'édition électronique : rôles des médiateurs et nouveaux rapports au savoir", dans le cadre du $70^{\mathrm{e}}$ Congrès de l'ACFAS «Publications et lectures numériques: problématiques et enjeux ", IEP Grenoble, 14 mai 2002.

16. Dans Libération du 13 novembre 2005, Patrick Bazin indique : "L'avenir des bibliothèques sera moins de jouer le rôle de sanctuaire de documents physiques que celui de passerelle, de médiateur de l'information ".

17. Perriault Jacques, La logique de l'usage, Paris, Flammarion, 1989.

18. Alter Norbert, L'innovation ordinaire, P.U.F. sociologies, Paris, 2000.

19. Bélisle Claire, Contrats de lecture, Rapport sur une expérimentation de prêt de livres électroniques en bibliothèques: dimensions technico-économiques et socio-cognitives, Lyon, 2002. Voir également: Claire Bélisle, "Colloque Les défis de la publication sur le web, Hyperlectures, cybertextes et méta-éditions », Lyon, 2002.

20. http://www.lib.rochester.edu/main/ebooks/studies/conclusions.htm

21. Bélisle Claire et Ducharme Christian, BBF 2003 - Paris, t. 48, n 3, pp. 74-86.

22. Newbiz, « Alexandre Jardin, écrivain », 21 juin 2000.

23. Morel Christian, Les décisions absurdes: sociologie des erreurs radicales et persistantes, Paris, Gallimard, 2002.

\section{RÉSUMÉS}

Cytale fut la société française conceptrice du livre électronique cybook, commercialisé en 2001 et 2002. Passée cette époque pionnière, et à l'heure où Sony exporte son Librié en Europe et aux États-Unis, cet article propose une analyse rétrospective pour comprendre la nature de la proposition de lecture que fit Cytale et préciser la filiation avec le livre papier que le cybook revendiquait; pour expliquer les termes et les conséquences des accords que Cytale scella, en précurseur, avec les éditeurs français; enfin pour évaluer la légitimité de son innovation, notamment aux yeux des différents publics cibles auxquels elle s'est adressée. Il est fait appel aux outils de la sociologie de la traduction, pour comprendre comment l'identité d'un objet technique, le Cybook, s'est construite, et comment ses fonctions lui ont été assignées ou refusées.

In 2001 and 2002, the French company Cytale manufactured and marketed an electronic book reader known as the Cybook. Four years later and at a time when Sony introduced its Librié on the European and American markets, this paper presents a retrospective analysis in order to understand the nature of Cytale's device, which consisted of reading digitally and electronically, to specify what kind of filiation with the paper book the Cybook was claiming, and to explain how Cytale set the seal on an agreement with French publishers as well as the consequences of this association. Finally, the paper evaluates the legitimacy of this innovation, notably from the point of view of the customers targeted by Cytale. The tools offered by the sociology of translation allow us to understand how the identity of a technical object-the Cybook-was constructed and how Cytale and its partners assigned (or denied) this e-book reader its functions. 
INDEX

Mots-clés : livre électronique, innovation, édition, sociologie de la traduction, stratégie commerciale, lecture

Keywords : innovation, electronic book, publishing, sociology of translation, commercial strategy, reading

\section{AUTEUR}

\section{DOMINIQUE NAUROY}

Dominique Nauroy est docteur en sciences de l'information et de la communication et coordinateur de projets multimédia. Il a soutenu sa thèse de doctorat en décembre 2005 : L'échec du livre électronique de Cytale au prisme des processus de traduction, mention très honorable avec les félicitations du jury. Ce travail, dirigé par le Professeur Jacques Walter, de l'université Paul Verlaine - Metz, est une contribution à l'analyse de la dynamique des innovations et de leur diffusion dans des sphères d'usages. Cette étude sera publiée au printemps 2007 aux presses de l'Enssib, dans la collection « Références ». Adresse électronique : dominique.nauroy@eleven.lu. 